\title{
An Analysis for the application of rapid rehabilitation surgery concept in the nursing satisfaction of the perioperative period of the elderly's hip arthroplasty
}

\author{
Shi Hua-ping \\ Hangzhou Fuyang Traditional Chinese Medicine Orthopaedic Hospital 311400 \\ https://orcid.org/0000-0002-4487-5920
}

Received: 12 December 2019 Accepted: 25 December 2019 Published: 31 March 2020

Cite this Article Shi Hua-ping. An Analysis for the application of rapid rehabilitation surgery concept in the nursing satisfaction of the perioperative period of the elderly's hip arthroplasty [J].Medical Research,2020.2(1): 21-25, http://dx.doi.org/10.6913/MRHK.202003_2(1).0005

Copyright (C) 2020 Creative Publishing Co., Limited. All rights reserved. Email: kycbshk@gmail.com.

\begin{abstract}
Objective To explore the application of rapid rehabilitation surgery concept in the nursing satisfaction of the perioperative period of the elderly's hip arthroplasty.

Methods To select 58 cases of elderly patients undergoing hip arthroplasty in our hospital from March 2017 to February 2018 as the research subjects. The patients were randomly divided into the experimental group and the control group by number table method and there were 29 cases per group. The patients in the experimental group were in the nurse of the concept model of rapid rehabilitation surgery, while the patients in the control group were in the routine nurse of perioperative period.
\end{abstract}

Results After the comparison of hip joint function recovery score, operation time and hospitalization time between the two groups of patients, the advantages of hip function recovery score, operation time, and hospitalization time in the experimental group were obvious statistically significant $(\mathrm{P}<\mathrm{UNK}>0.05)$. Compared with the patients in the control group, the nursing satisfaction of the patients in the experimental group was higher and statistically significant $(\mathrm{P}<\mathrm{UNK}>0.05)$.

Conclusion The concept of rapid rehabilitation surgery is effective for the elderly patients with hip arthroplasty, which can promote the rehabilitation of patients and improve the nursing satisfaction of patients. It is of great significance to improve the relationship between nursing patients.

Key words the concept of rapid rehabilitation surgery; elderly; hip arthroplasty; nursing satisfaction

Hip arthroplasty is a commonly used clinical treatment method in orthopaedic surgery. Artificial prostheses containing femoral and acetabular parts are fixed to the patient's normal 
bone by cement and screws to replace the affected joint, so that the patient's hip joint function can be restored. It is currently a mature surgical program[1]. Due to the degenerative changes in all physiological functions of elderly patients, such as osteoporosis accompanied by some elderly patients accompanied, etc., they result in a relatively high probability of joint injury in patients. The effective treatment and care promote the rehabilitation of elderly patients and improve the patients' prognosis. This paper is mainly to explore the concept of rapid rehabilitation surgery applying the nursing satisfaction of the perioperative period of hip arthroplasty in the elderly. The findings are reported below.

\section{INFORMATION AND METHODS}

\subsection{General information}

In this experimental study, there were 58 elderly patients treated with hip arthroplasty in our hospital were analyzed and the time of the experimental subjects selection was from March 2017 to February 2018. The group was grouped according to the random number table method. The number of patients in the experimental group was 29, while that in the control group was 29. The patients in the experimental group were in the nurse of the rapid rehabilitation surgical model and the patients in the control group were in the perioperative routine nursing. Among the patients in the experimental group, there were 15 male patients and 14 female patients. The patient's age was $60-83$ years old. The average age was $(71.37 \pm 3.28)$ years old, in which 3 patients were osteonecrosis of the femoral head (ONFH), 2 patients were hip osteoarthritis, 5 patients were intertrochanteric fractures of the femur and 19 were femoral neck fractures. Among in the control group, there were 14 male patients, 15 female patients and their age of the patients was 59-84 years old, with an average age of $(71.99 \pm 3.64)$ years old, of which 3 patients were osteonecrosis of the femoral head(ONFH), 3 patients were hip osteoarthritis, 6 patients were intertrochanteric fractures and 17 patients were femoral neck fractures. After comparison of the general information of the experimental group and control group, their age difference is not significant and there is no statistical significance $(P>0.05)$.

\subsection{Methods}

The patients in the control group were given perioperative routine nursing for the guidance of preoperative examinations and health education. After the operation, observe the physiological indexes of the patients closely and instruct the patients to take early rehabilitation training to promote the recovery of the patients. The patients in the experimental group were given the nurse of concept mode of rapid rehabilitation surgery: (1) Preoperative Evaluation: Know about the patients' condition, inquire the general information such as disease history and allergy history and give a guidance of relevant preoperative examinations according to patients' assessment.(2) Preoperative Health Education: Preoperative health education should be given to patients and their families. Inform patients' families and their related conditions and inform the patients and their families' about relevant treatment methods and prognosis. Explain the possible risks during operation and the main points and complications of post-operative nursing, so that patients and their families can make corresponding psychological preparations. After explaining successful cases, the tension has been alleviated, the importance of patients' cooperation with treatment has been informed, so that patients' treatment determination can be enhanced to actively cooperate with treatment. Preoperative fasting and water deprivation should be maintained for 6 hours.(3) Preoperative Analgesia: Preoperative analgesia can 
effectively alleviate the pain of orthopaedic patients after operation and reduce the dosage of analgesics, so that patients' emergency response can be reduced and treatment satisfaction can be improved. (4) Preoperative Exercise: Nurses should guide patients to carry out breathing exercises before operation, enhance patients' lung function and carry out limb and ankle pump exercise and quadriceps femoris training for patients, laying a foundation for rehabilitation training after operation. (5) Postoperative Care: After completion of the operation, get close observation of the patient's physiological index, if the patient's blood pressure, body temperature and other index were found abnormal, report to the doctor for treatment in time. (6) Antithrombotic Nursing Care: Nurses should regularly massage the lower limbs and healthy limbs of patients to promote blood circulation, prevent the formation of deep vein thrombosis of lower limbs and reduce the occurrence of complications. (7) Dietary Care: Patients are advised to eat high protein, vitamins, dietary cellulose content food and remember to drink water, as a large number of water can effectively prevent urinary tract infection and other complications.

\subsection{Observation Index}

The hip function recovery score, operation time, hospitalization time and nursing satisfaction were compared between the two groups. The patients' hip function score was judged by Harris score, with a total score of 100 . The higher the scores is, the better the recovery effect of the patients have. Nursing Satisfaction $=$ Satisfaction Rate + Very Satisfaction Rate

\subsection{Statistical Analysis}

All the data of 58 patients in this experiment were processed by SPSS17.0 software. The hip function recovery score, operation time and hospitalization time were expressed in the form of mean (+standard deviation), t-test, nursing satisfaction ratio (\%) and chi-square test. When the information comparison showed the difference of $\mathrm{P}<0.05$, there was a statistical significance.

\section{RESULTS}

\subsection{Comparison of hip function recovery score, operation time and hospitalization time between two groups}

The hip function recovery score, operation time and hospitalization time of the experimental group were significantly better than those of the control group $(\mathrm{P}<0.05)$, with a different statistics significance as shown in Table 1.

\begin{tabular}{|c|c|c|c|c|}
\hline Group & $\mathrm{n}$ & $\begin{array}{c}\text { hip function recovery } \\
\text { scores/points }\end{array}$ & Operation time/min & $\begin{array}{c}\text { Hospitalization } \\
\text { time } / \mathrm{d} \\
\end{array}$ \\
\hline Experimental group & 29 & $92.64 \pm 3.24$ & $126.35 \pm 10.21$ & $17.64 \pm 3.69$ \\
\hline Control group & 29 & $84.11 \pm 3.65$ & $145.57 \pm 15.24$ & $27.37 \pm 1.24$ \\
\hline $\mathrm{t}$ & - & 9.4118 & 5.6423 & 13.4602 \\
\hline$P$ & - & 0.0000 & 0.0000 & 0.0000 \\
\hline
\end{tabular}

2.2 Comparison of nursing satisfaction between experimental group and control group The nursing satisfaction of the experimental group was significantly higher than that of the control group $(\mathrm{P}<0.05)$, with a statistics significance as shown in Table 2. 
Table 2 Comparison of nursing satisfaction between experimental group and control group [n (\%)]

\begin{tabular}{cccccc}
\hline Group & $\mathrm{n}$ & Very satisfaction & Satisfaction & Non satisfaction & Nursing satisfaction \\
\hline Experimental group & 29 & 16 & 12 & 1 & $28(96.55)$ \\
Control group & 29 & 12 & 14 & 6 & $26(89.66)$ \\
$\mathrm{X}^{2}$ & - & - & - & - & 4.0616 \\
$\mathrm{P}$ & - & - & - & - & 0.0438 \\
\hline
\end{tabular}

\section{DISCUSSION}

Hip arthropasty is an effective way to treat hip diseases such as hip fracture. Damaged hip joint can affect the daily activity of patients and reduce the quality of life. With the continuous improvement of people's living standards, there is a higher demand for the quality of nursing. The concept of rapid rehabilitation surgery is a scientific nursing mode. In clinical practice, this nursing mode is often applied to patients undergoing surgical treatment. For the elderly patients with hip replacement, this nursing mode can effectively reduce the pain of patients and promote the rehabilitation of patients.

Rapid rehabilitation surgery nursing concept pays more attention to preoperative education for patients, so that patients and their families can face up to the disease, and effectively alleviate the pain of patients through preoperative use of analgesics, reduce the dosage of analgesics and complications, so that patients have a good mentality to accept surgery, and actively cooperate with doctors for treatment. During the operation, patients need to complete the assistant operation according to the doctor's requirements. After the operation, the patient's physiological indexes were monitored dynamically. Once abnormalities occur, they can be effectively handled. Dietary nursing can promote the patient's reasonable diet, provide the necessary energy for the body's daily activities, and enhance the patient's immunity [4]. The dietary nursing can protect the patients' intestinal mucosa and promote the circulation of portal vein, accelerate the functional recovery of various organs to a certain extent. After the limb rehabilitation training, if patients have stable surgical conditions, they can exercise lower limbs and hands in the hospital bed, so as to promote blood circulation, reduce the occurrence of complications such as thrombosis, pressure sore, etc. To a certain extent, they shorten the hospitalization time of patients, guaranteeing the patients' therapeutic effect and promoting patients' rehabilitation [5]. Postoperative rehabilitation exercise is an important part of postoperative nursing care of hip joint. In the early rehabilitation, patients need to exercise step by step, so as to prevent secondary injury. Effective rehabilitation training can improve patients' blood and lymphatic circulation, promote absorption, reduce pain feeling and promote rehabilitation[6].

To sum up, the concept of rapid rehabilitation surgery has a remarkable effect in perioperative nursing of elderly patients with hip arthropasty. It has the characteristics of short operation time and short hospitalization time. It can improve patients' nursing satisfaction and promote the recovery of hip nerve function. This kind of nursing method is worth popularizing in clinical practice.

\section{REFERENCES}

[1]YANG Wei, YANG Liu, CHEN Hong. Progress of the application of rapid rehabilitation surgery concept in the perioperative nursing care of elderly hip arthroplasty surgery[J]. Laboratory Medicine and 
Clinic , 2014,29(24): 3499-3500.

[2]LIU Wei, XU Fengjuan, ZHUANG Meihua. Application of rapid rehabilitation surgery concept in perioperative period of elderly patients' hip arthroplasty surgery [J]. Chinese Journal of Trauma and Disability Medicine, 2016, 24(24): 14-16.

[3] Liang Jianmin. Application of rapid rehabilitation surgery in perioperative period of elderly patients' undergoing hip arthroplasty surgery[J]. Chinese General Nursing, 2017, 15(4): 436-437.

[4]Chen Xueli. Application of rapid rehabilitation surgery concept in perioperative nursing care for elderly patients' hip arthroplasty surgery[J]. Medicine and People, 2014,34(11):30-30.

[5] Liu Fuguojie, Yan Jun, Zhu Guohua. Application of rapid rehabilitation surgery concept in perioperative period of elderly patients' undergoing hip arthroplasty surgery[J]. Clinical Medical \& Engineering, 2018,6(4)25.

[6]XIONG Jianqiu, LI Yuhua, DING Li. Application of rapid rehabilitation surgery concept in postoperative nursing care for elderly patients undergoing hip arthroplasty surgery[J]. Journal of Nursing, 2014, 16(5): 43-44. 